\title{
Experience with a fluorescence-based beam position monitor at the APS
}

\author{
E.M. Dufresne*†, J.A. Guzman*, S B. Dierker**, R. Clarke*, D.A. Arms ${ }^{\dagger}$ and D.A. \\ Walko $^{\dagger}$
}

\author{
* Department of Physics, University of Michigan, Ann Arbor MI 48109 \\ ${ }^{\dagger}$ MHATT-CAT, Advanced Photon Source, Argonne National Lab., Argonne IL 60439 \\ ** also at the National Synchrotron Light Source, Brookhaven National Lab., Upton NY 11973
}

\begin{abstract}
We have recently redesigned the first crystal mount of our cryogenically cooled monochromator to reduce its sensitivity to pressure fluctuations in the cryogenic lines feeding the Si (111) crystal. With the use of a fluorescence-based X-ray beam position monitor (BPM) placed $19 \mathrm{~m}$ away from the monochromator, much operational experience has been gained on the sensitivity of the beam position and intensity to small changes in the cooling system. In this presentation, we will describe our X-ray BPM design and performance and will provide examples of changes that have made the beam position more stable on our beamline. One such change for example has been the top-up operation of the Advanced Photon Source (APS), which has reduced the thermal drifts associated with the ring current decay.
\end{abstract}

Novel experiments at the Advanced Photon Source (APS) typically require a very stable beam [1,2]. Being able to know the position of the X-ray beam while conducting experiments at synchrotron radiation sources can be a very valuable information. For example, one can use feedback to keep the position constant, or realign the beamline when significant changes have been noticed on the beam position monitor (BPM). At the APS, the source point typically moves with a standard deviation of $4 \mu \mathrm{m}$ and $2 \mu \mathrm{m}$ in the horizontal and vertical direction, respectively. Furthermore, the typical X-ray beam pointing stability is on the order of $0.1 \mu \mathrm{rad}$. Thus, for an experiment $60 \mathrm{~m}$ from the source, beam motion on the order of $10 \mu \mathrm{m}$ is expected during normal operation.

We have recently designed a high vacuum (HV) BPM which uses the X-ray back fluorescence from a metal foil, such as $\mathrm{Cr}$ or Ti, detecting it by a simple quadrant photodiode array, resulting in a device with beam position resolution on the order of one micron [3]. The BPM is a modification of a well-tested BPM design [4]. Each photodiode accepts a solid angle that is sensitive to the beam position on the foil. For a small displacement in the range of a few $\mathrm{mm}$, the ratio of the two vertical diode currents $i_{y 1}$ and $i_{y 2}$, for example, $R_{y}=\left(i_{y 1}-i_{y 2}\right) /\left(i_{y 1}+i_{y 2}\right)$, is proportional to the displacement $\Delta y$ from the center of the diode array [4].

The device is permanently installed on beamline 7ID, $19.2 \mathrm{~m}$ from the cryogenic monochromator [5]. A detailed construction report can be found elsewhere [3]. The main differences found in our version of the Alkire et al. [4] BPM are that the diode holder is made with G10, a fiberglass composite material compatible with a vacuum environment, and that the upstream side of the diodes is shielded with a 1-mm-thick Ta plate. The geometry used is the same as that described in Ref. [4]. The diodes and foil are held by the G10 mount inside a five-way vacuum cross, itself mounted on an X-Y motorized translation stage. The signals from the four diodes are amplified by an ON-TRAK model OT-301 position-sensing amplifier which outputs three separate signals, i.e., a signal proportional to the sum of the four diode currents, and two signals varying from -10 to $10 \mathrm{~V}$, proportional to the vertical and horizontal ratios $R_{x}$ and $R_{y}$. This commercial amplifier has six ranges of transimpedance gains with the highest gain set to $4 \times 10^{6} \mathrm{~V} / \mathrm{A}$. We have found it works well with beams from an insertion device beamline at the APS. These three signals are digitized by a 16 bit $\mathrm{A} / \mathrm{D}$ board. The calibration of the diodes can be done by scanning the $\mathrm{X}-\mathrm{Y}$ translation stage and recording the digitized ratios.

It can be easily shown that the current on one of the diodes generated by the X-ray fluorescence is given by

$$
i=\alpha\left(\frac{Y(1-T) \Phi}{4 \pi} \frac{E_{C r}}{E_{i}} e\right) \int_{\Omega} d \Omega, \text { where } \int_{\Omega} d \Omega=\int_{-\phi}^{\phi} \int_{\theta_{1}}^{\theta_{2}} \sin d \theta d \phi=[\phi]_{-\phi}^{\phi}[-\cos \theta]_{\theta_{1}}^{\theta_{2}} .
$$



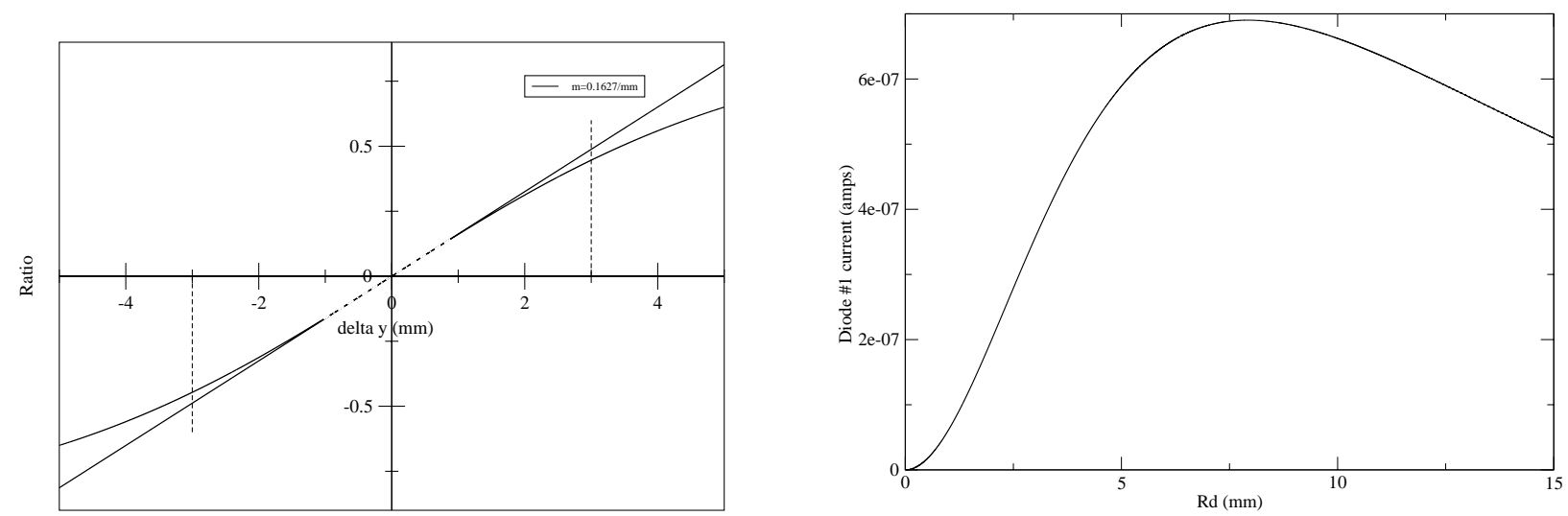

FIGURE 1. (left) The theoretical calibration ratio vs $\Delta y$. (right) The estimated current from a simple theory for the Cr case described by Equation 1.

In Equation 1, $\alpha=100 \%$ is assumed for the quantum efficiency of the detectors, $Y$ is the fluorescence yield for $\mathrm{Cr}$ (28\%), $T$ is the transmission of the $\mathrm{Cr}$ foil at $10 \mathrm{keV}(95.5 \%), \Phi=1.0 \times 10^{13}$ photons/sec is the flux of the $\mathrm{x}$-ray beam, $E_{C r}=5415 \mathrm{eV}$ is the $\mathrm{Cr} \mathrm{K} \alpha$ energy, $E_{i}=3.55 \mathrm{eV}$ is the binding pair energy of $\mathrm{Si}$, and $e$ is the electron charge.

The vertical beam position is determined from the ratio

$R_{y}=\frac{i_{1}-i_{2}}{i_{1}+i_{2}}=\frac{\cos \theta_{1}-\cos \theta_{2}-\cos \theta_{3}+\cos \theta_{4}}{\cos \theta_{1}-\cos \theta_{2}+\cos \theta_{3}-\cos \theta_{4}}=\frac{\frac{Y_{d}-\Delta y+L}{\sqrt{R_{d}^{2}+\left(Y_{d}-\Delta y+L\right)^{2}}}-\frac{Y_{d}-\Delta y}{\sqrt{R_{d}^{2}+\left(Y_{d}-\Delta y\right)^{2}}}+\frac{\left(Y_{d}+\Delta y\right)}{\sqrt{R_{d}^{2}+\left(Y_{d}+\Delta y\right)^{2}}}-\frac{\left(Y_{d}+\Delta y+L\right)}{\sqrt{R_{d}^{2}+\left(Y_{d}+\Delta y+L\right)^{2}}}}{\frac{Y_{d}-\Delta y+L}{\sqrt{R_{d}^{2}+\left(Y_{d}-\Delta y+L\right)^{2}}}-\frac{Y_{d}-\Delta y}{\sqrt{R_{d}^{2}+\left(Y_{d}-\Delta y\right)^{2}}}-\frac{\left(Y_{d}+\Delta y\right)}{\sqrt{R_{d}^{2}+\left(Y_{d}+\Delta y\right)^{2}}}+\frac{\left(Y_{d}+\Delta y+L\right)}{\sqrt{R_{d}^{2}+\left(Y_{d}+\Delta y+L\right)^{2}}}}$,

where $R_{d}=8.89 \mathrm{~mm}$ is the distance foil-diodes; $Y_{d}=6.707 \mathrm{~mm}$, the distance from the center line to the bottom of the photodiode; $L=9.7 \mathrm{~mm}$, the active length of the photodiode; and $\Delta y$ is an arbitrary change in beam position. The angles $\theta_{1-4}$ in Equation 2 are the angle originating from a point source on the foil plane subtended by the diodes corresponding to the vertical limits of the top and bottom diodes. This calculation for the X-axis is identical. The calibration curve of the device is the derivative of the ratio $\mathrm{R}$ versus $\Delta y$. Taking a derivative of Equation 2, one finds

$$
\lim _{\Delta y \rightarrow 0} \frac{R(\Delta y)-R(0)}{\Delta y}=0.1627 / \mathrm{mm}
$$

The predicted sensitivity of the device for $\Delta y \ll 1$ agrees well with the measurement in Ref. [4]. Figure 1 (left) shows this slope as the straight line. The dashed line in the center is where the sensitivity is within $1 \%$ of the $0.1627 / \mathrm{mm}$ line, and the outer dashed line extending to the graph shows the $10 \%$ range of sensitivity.

Equation 2 is only dependent on the solid angle of the diodes. As one brings the photodiodes closer to the foil, at a certain point the angle between the photodiodes surface normal and the fluorescence direction vector approach a right angle, thus the signal goes to zero in Figure 1 (right). The predicted current in Figure 1 peaks at $R_{d}=7.921 \mathrm{~mm}$ with $6.903 \times 10^{-7} \mathrm{~A}$. The value chosen for this device was $R_{d}=8.89 \mathrm{~mm}$. This was chosen because the slope where $R_{d}<7.921 \mathrm{~mm}$ is much steeper than the slope when $R_{d}>7.921 \mathrm{~mm}$, thus errors in machining are less sensitive for the latter case.

Figure 2 (left) shows the digitized output X-ratio and Y-ratio of the OT-301 versus the X position of the X-ray BPM, which is scanned $\pm 1 \mathrm{~mm}$. The gain of the amplifier was set to $4 \mu \mathrm{A} / \mathrm{V}$. The $\mathrm{X}$ ratio varies linearly with a slope of -1.14 $\mathrm{V} / \mathrm{mm}$. Some slight cross-talk is observable on the Y-ratio but is fairly small. Scanning the Y position of the X-BPM shows a similar behavior with a slope of $-1.22 \mathrm{~V} / \mathrm{mm}$. The difference in slope is believed to originate from the different beam size in the vertical and horizontal direction [4]. The crosstalk may be caused by an imperfect alignment of the diode array to the motorized XY stage.

Figure 2(right) shows the calibrated beam position in a half-hour-long time series, with data points taken every second. The beam is stable to about $1 \mu \mathrm{m}$ root-mean-square (RMS) in both directions. The noise exhibits some correlation with fast rise time and longer decay time, which we attribute to the closed-loop heater turning on every 55 seconds or so [5]. A time correlation function of the X-position fluctuations fit relatively well an exponential decay 

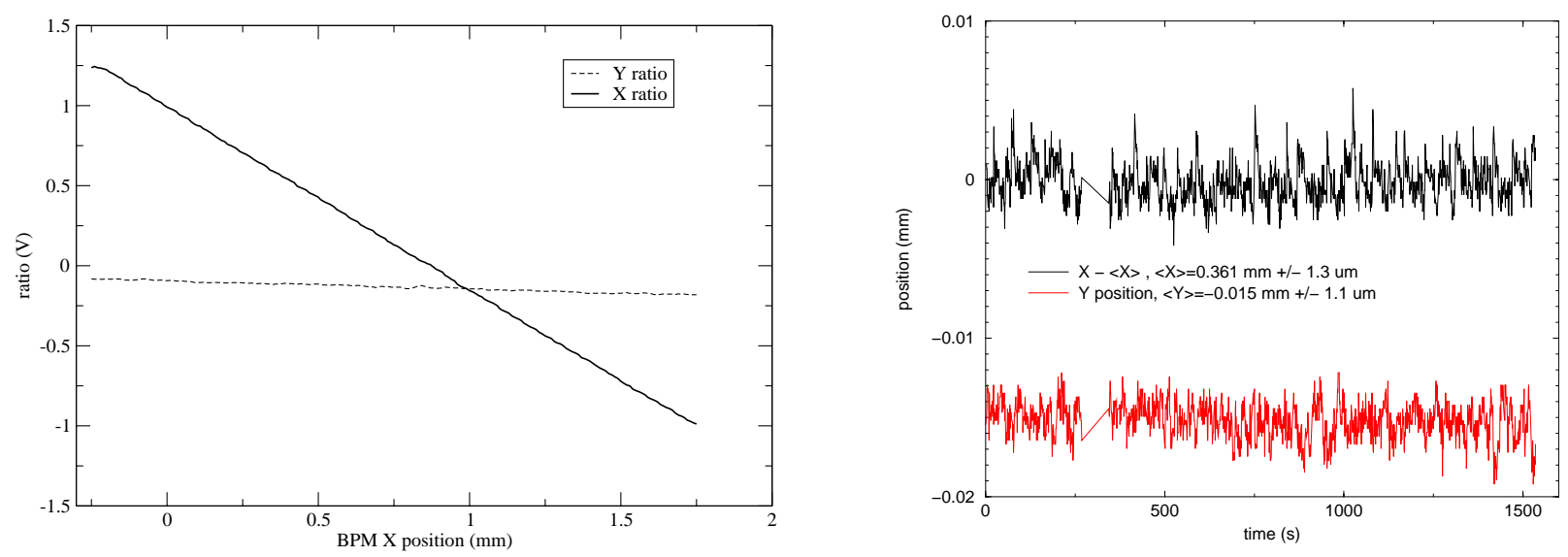

FIGURE 2. (left)The horizontal calibration at $10 \mathrm{keV}$ with nearly the full 7ID central cone incident on the foil. (right) A short time series showing the sensitivity of the device.

with a $3.9 \mathrm{~s}$ correlation time (see Figure 3 (left)). Clearly we can resolve these $1-5 \mu \mathrm{m}$ beam position fluctuations since they are significant when studied by a correlation analysis.
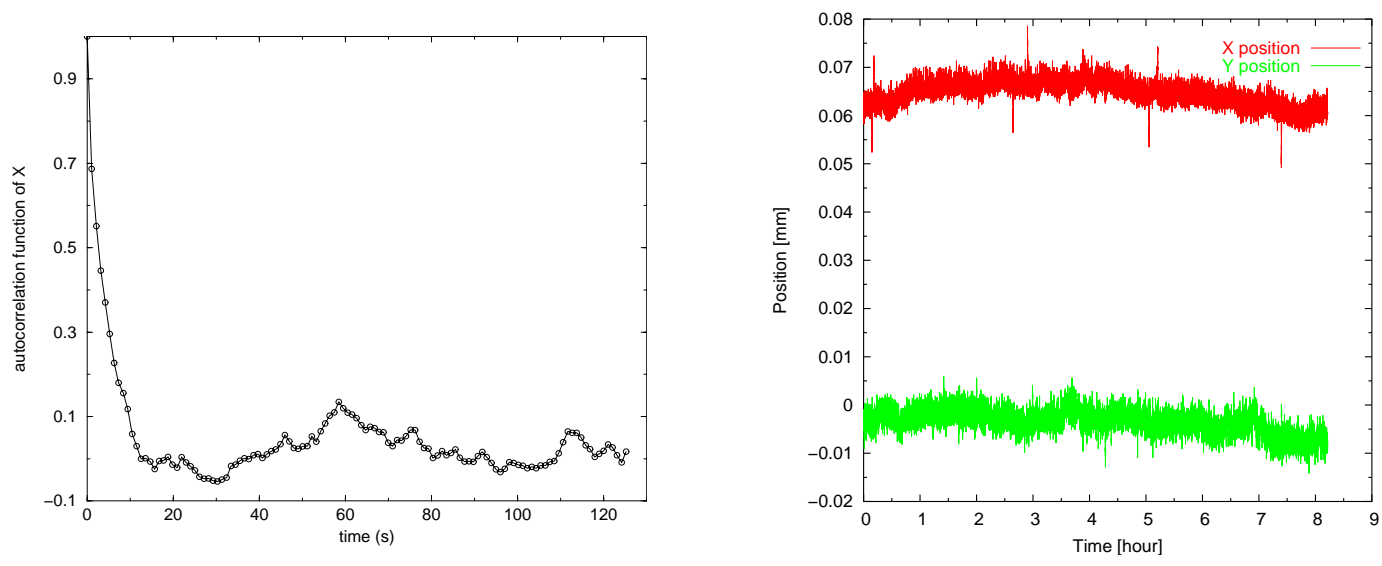

FIGURE 3. (left)The time correlation of the X-position in Figure 2. (right)The beam position in a time series measured during top-up mode.

In Ref. [5], we discussed the changes made to the first crystal mount of the cryogenically cooled monochromator but did not show directly the improvements in beam position stability. Figure 3 (right) shows the beam position measured in top-up mode in a time series started $11 / 17 / 01$ at $2 \mathrm{~h} 17 \mathrm{am}$. A white beam with a fundamental set to $12.5 \mathrm{keV}$ and apertured by $0.5 \mathrm{~mm} \times 0.5 \mathrm{~mm}$ was incident on the monochromator, itself set to diffract $12.5 \mathrm{keV} \mathrm{X}$-rays. The beam is stable well within $10 \mu \mathrm{m}$ in these data. We have found that top-up operation has stabilized our monochromatic beam due to the constant heat load. Although the Compton shielding inside the monochromator is poor, after a warm-up period, the beam remains stable within tens of micron in top-up operation.

Figure 4(left) shows a time series of the diode sum, ring current, and an ion-chamber signal in the upstream hutch 7ID-B. The undulator fundamental was set to $9.06 \mathrm{keV}$, the monochromator Bragg angle was set to diffract $9.0 \mathrm{keV}$ $\mathrm{X}$-rays, and the white beam slit before the monochromator was set to $1.0 \mathrm{~mm}(\mathrm{H})$ by $0.5 \mathrm{~mm}(\mathrm{~V})$. The time series started on 10/19/2002 near midnight and lasts a day and a half until the beam dumps. A top-up failure occurred 13 hours into the time series as seen in the ring current dip, and the signals in all the detectors shown are correlated to this dip. The 7ID-B ion chamber and the 7ID-C diode sum overlap well with the ring current, thus the diode sum can serve as an additional beam intensity monitor. Although top-up reduces the thermal drifts due to the change of current in the machine, it also introduces time fluctuations of its own every 2 minutes when the ring is refilled. These explain the fast changes in the ring current in Figure 4(left). 

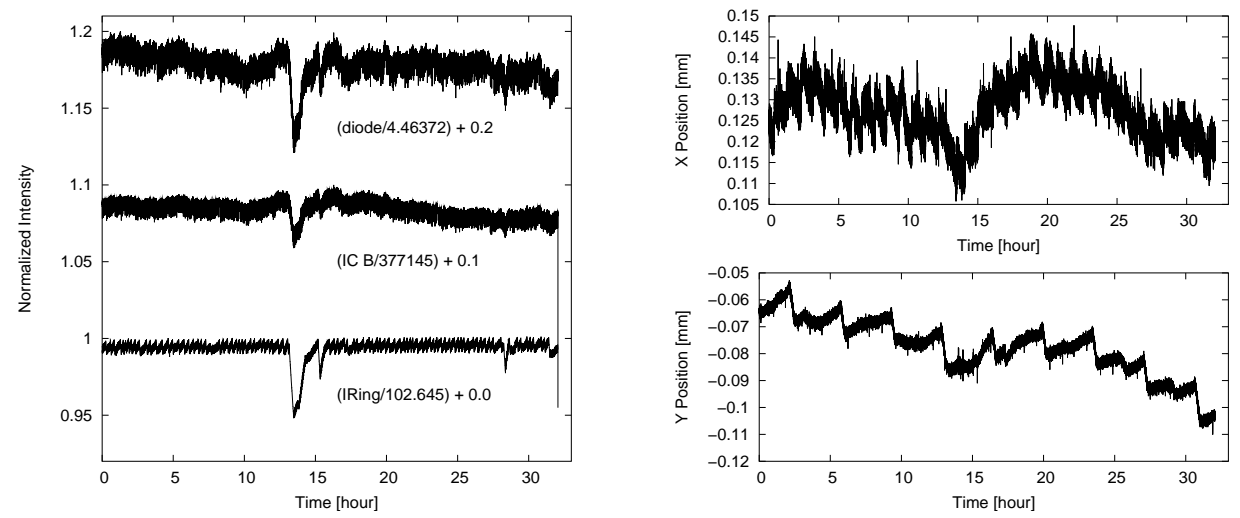

FIGURE 4. (left) The diode sum, ring current and 7ID-B ion-chamber signal versus time during top-up operation. (right) The beam position in the same time series.

Figure 4(right) shows the 7ID-C BPM signals. In the vertical, apart from a drift of $30 \mu \mathrm{m}(1 \mu \mathrm{m} /$ hour $)$ noted above, bumps are seen every 3.5 hours due to the LN2 cryocooler fill. Thirteen hours into the time series, the top-up failure causes a bump in both $\mathrm{X}$ and $\mathrm{Y}$ positions. A long time-scale oscillation with a period greater than 12 hours is seen in the $\mathrm{X}$ position. Since the APS was then running in 23 bunch mode, it takes on average a little longer than 46 minutes to refill all the buckets. When the bucket used to measure the electron beam position is refilled, the change of current of this bunch causes a perceived beam motion on the X-ray radio frequency BPM, which is then corrected by the feedback system. This explains the horizontal beam motion measured by the diode system every hour or so. The APS has implemented an improvement in the BPM timing system that samples all bunches, significantly reducing this source of beam motion [6].

The BPM has been tested with a small $(0.1 \mathrm{~mm})^{2}$ pink beam, and the foil survived several weeks of operation in this mode. We also have used the BPM to calibrate the angular motion of our second monochromator crystal piezoelectric rotation actuator. Our second-crystal $\chi$ angle is controlled by a picomotor, which is not a very reproducible and scannable actuator. Because of the installation of the BPM, steering the beam in the horizontal direction with this picomotor is now straightforward and reproducible. This BPM design provides an inexpensive and versatile detector that has greatly enhanced the operation of the 7ID beamline.

\section{ACKNOWLEDGMENTS}

We wish to thank Nino Pereira for his help during the installation of the BPM. This work was done at the MHATT-CAT 7ID beamline, and was supported in part by DOE Grant No. DE-FG02-03ER46023 and DE-FG02-00ER15031, and by the NSF FOCUS physics frontier center. Use of the Advanced Photon Source is supported by the U.S. Department of Energy, Basic Energy Sciences, Office of Energy Research, under Contract No. W-31-109-ENG-38.

\section{REFERENCES}

1. Yacoby, Y., Sowwan, M., Stern, E., Cross, J., Brewe, D., Pindak, R., Pitney, J., Dufresne, E., and Clarke, R., Nature Materials, 1, 99-101 (2002).

2. Dufresne, E. M., Nurushev, T. S., Dierker, S., and Clarke, R., Phys. Rev. E, 65, 061507-1-061507-9 (2002).

3. Guzman, J., A UHV X-ray beam positioning system, using a quadrant PIN photodiode array (2001), available on www. mhatt.aps.anl.gov/research/publications/reports/.

4. Alkire, R., Rosenbaum, G., and Evans, G., J. Synch. Rad., 7, 61-68 (2000).

5. Dufresne, E. M., Arms, D., Dierker, S., Clarke, R., Yacoby, Y., Pitney, J., MacHarrie, B., and Pindak, R., Rev. Sci. Instrum., 73, 1511-1513 (2002).

6. Lenkszus, F., Laird, R., Singh, O., and Decker, G., "New APS Storage Ring BPM Timing System Design," in Proceedings of the 2001 Particle Accelerator Conference, Chicago, IL, 2001, available at http://accelconf.web.cern.ch/accelconf/p01/PAPERS/MPPH001.PDF. 